\title{
KASABACH-MERRIT syndrome triggered by vaccination
}

\author{
${ }^{1}$ S. Batali ${ }^{*},{ }^{2}$ H. Aguenaou, ${ }^{3}$ A. Barkat \\ ${ }^{1}$ Department of Neonatology in Rabat children's hospital, \\ ${ }^{2}$ Faculty of medicine and pharmacy in Rabat, \\ ${ }^{3}$ Mohamed V University / Ibn Tofail University
}

\begin{abstract}
The Kasabach-Merritt syndrome is a rare disorder of the newborn or very young child which combines a rapidly expanding red-purple vascular tumor and a hematological syndrome dominated by major thrombocytopenia and more moderate consumption of fibrinogen and coagulation. This syndrome is always considered serious due to hemorrhages related to coagulopathy. Imaging confirms the diagnosis and searches for deep locations. Therapeutic management must be early, multidisciplinary, adapting the benefits to therapeutic risks in order to improve the prognosis. We report the case of a KASABACHMERRITT Syndrome on a preexisting thigh angioma triggered by vaccination in a female infant treated with angiogenesis SIROLIMUS, to focus on the cause and effect relationship between vaccination and SKM in the light Department of Neonatology in Rabat children's hospital, Faculty of medicine and pharmacy in Rabat, of literature.
\end{abstract}

Keywords: KASABACH-MERRITT syndrome, vaccination, SIROLIMUS.

\section{Introduction}

Kasabach Merritt syndrome (SKM) is defined by the association of a rapidly expanding red-purple vascular tumor, thrombocytopenia, varying degrees of disseminated intravascular coagulation (DIC) and sometimes anemia. It usually occurs in newborns and infants less than 6 months old, sometimes even in utero [1]. This syndrome deserves special attention because of its clinical, therapeutic and prognostic seriousness since it involves the vital prognosis with high mortality [2], [3]. We report a case of Kasabach-Merritt syndrome triggered by vaccination. We will deal with its various diagnostic and therapeutic difficulties and we will try, in the light of the literature, to understand the relationship between SKM and vaccination.

\section{Case Report}

RA infant 2 months 22 days old, female, of non-consanguineous Parents, unique in his family, of a well followed pregnancy with APGAR 10/10, presenting at birth a cutaneous hemangioma at the level of the right thigh evolving a few days after vaccination at the age of 2 months (2nd vaccine) towards a hematoma, reddish purple, warm, sensitive swelling on the skin and a smooth shiny surface strewn with a few bruises and petechiae, gradually increasing in volume (right thigh, right inguinal fold) figure 1

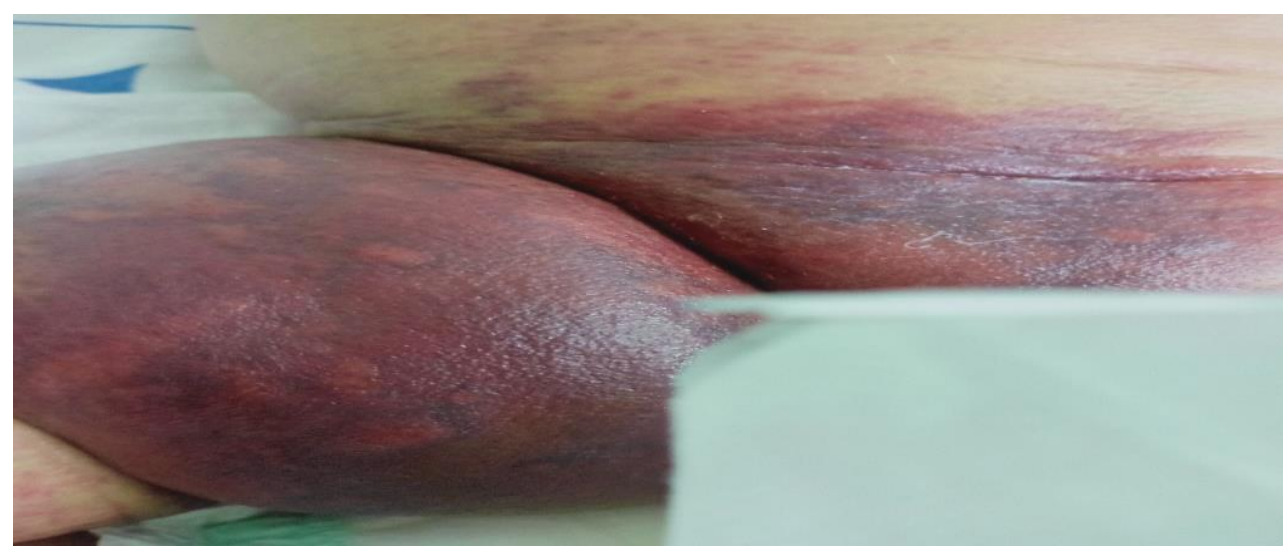

Figure 1: swelling of the right thigh in post-vaccination 
The rest of the somatic examination was normal. The biological assessment showed anemia (hemoglobin: $6.6 \mathrm{~g} / \mathrm{dl})$ with thrombocytopenia $(8,000 / \mathrm{mm} 3)$ and a collapsed PT. Doppler ultrasound of the right lower limb to objectified a hemangioma associated with a diffuse hematoma of the abdominal wall as well as the superficial planes of the whole of the right lower limb predominant at the level of the groin and the root of the thigh. Abdominal ultrasound, ETF, ETT and chest X-ray did not reveal any other associated abnormalities. The infant was then transfused with blood cells, platelet pellets and fresh frozen plasma, then put on a corticosteroid bolus 3 days with oral relay at a rate of $2 \mathrm{mg} / \mathrm{kg} /$ day for 4 weeks with depression over 6 months associated with beta-blocker and with acetylsalicylic acid at $10 \mathrm{mg} / \mathrm{kg} / \mathrm{d}$, the evolution was marked by the reduction in the size of the bruise but persistence of a bulky swelling not allowing surgical resection hence the initiation of treatment based on immunosuppressant (SIROLIMUS *) with stabilization of the clinical condition of our patient without any detectable adverse effect to date.

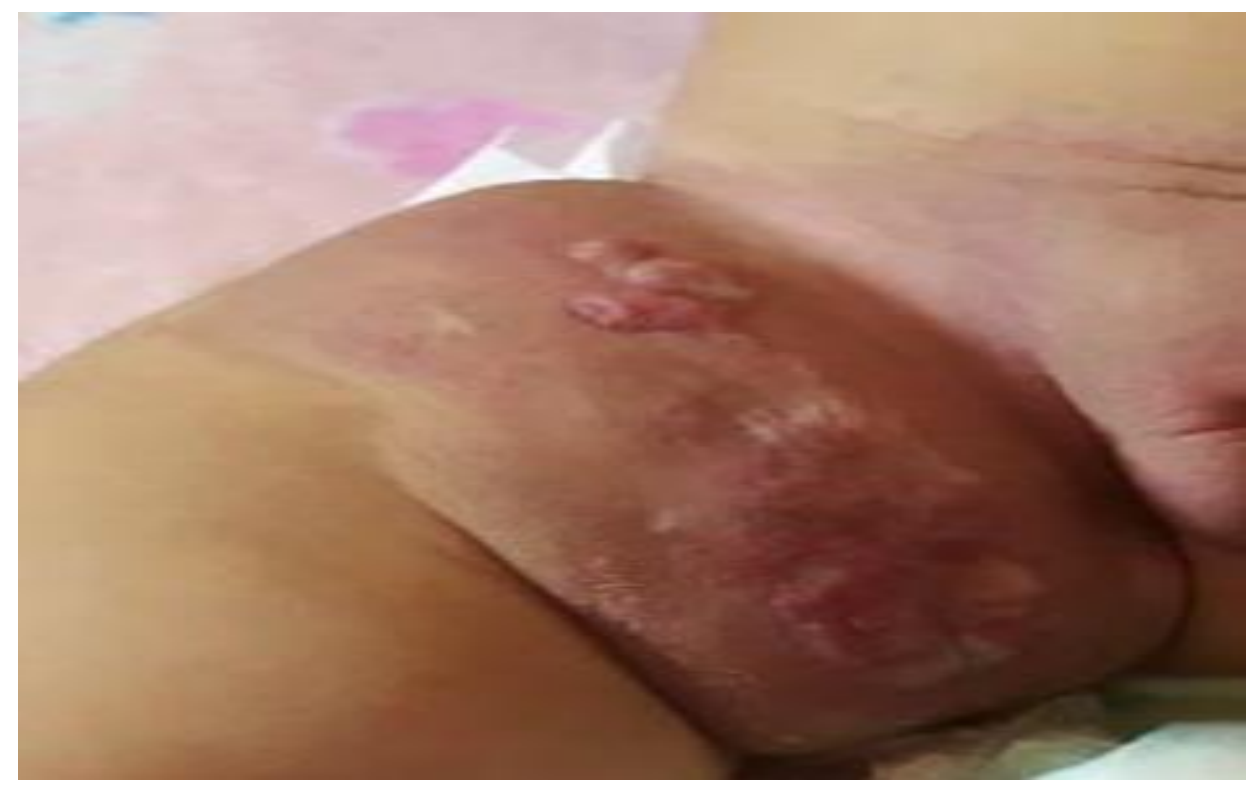

Figure 2: Appearance of swelling after the introduction of Sirolimus

\section{Discussion}

Since the first description in 1940, the SKM designated the association of a thrombocytopenia and a bulky hemangioma. Until 1997, it was considered a complication of classical childhood hemangiomas [1], [2]. Currently, it is characterized by a tumor which makes the bed of biological phenomenon sometimes lethal associating a thrombocytopenia, a DIC and sometimes an anemia [1], [2]. The hemangioma can be cutaneous sometimes associated with occult visceral hemangiomas of different localizations often abdomino-pelvic (hepatic, splenic, retro peritoneal), cervico-thoracic, mediastinal and osseous [1], [3], [4], [5] ] It combines a particular vascular lesion, often classified as immature hemangiomas, and a hematological syndrome dominated by major thrombocytopenia [1]. It is a rare syndrome that most often affects boys. The age of onset is early: $25 \%$ of Kasabach-Merritt syndromes are present in the neonatal period and $80 \%$ of cases before 3 months [2], its pathophysiology is currently better elucidated thanks to clinical pathological, biological and genetic advances; it is believed to be a platelet sequestration within the vessels, linked to an abnormal and proliferating endothelium [6, 7]. Various immunohistochemical markers of platelets have been used to confirm this sequestration, in particular the anti-CD 61 antibody [8]. This platelet sequestration would lead to platelet activation and consumption of the coagulation factors, which cause DIC. This could favor the occurrence of thromboses and intra-tumor hemorrhages [7]. A series of studies has shown that platelets are trapped in tumors. However, the trapping of platelets is not the same as the episode of SMP. Retrospective research has shown that there are no radiological or microscopic differences between non-coagulopathic KHE and coagulopathic KHE. Evidence of platelet trapping was seen histologically, despite normal serum platelet levels [9]. The KHE on the thigh of a 2 month old boy started to swell soon after the coxsackie viral infection [9]. A 15-day-old boy suffered from worsening angioma on his left cheek, associated with SKM, shortly after the first vaccination against the hepatitis B virus [10]. A retrospective examination of 107 patients showed that the risk of SKM increases considerably when the tumor infiltrates the muscle. Repetitive strain injury from muscle movement appears to contribute 
to SKM [11]. It appears that the structure of KHE can cause trapping of activated platelets, which could be aggravated by incentives, such as infection [9], vaccination [10] and trauma [11], which is the case with our patient.

The clinical appearance of the vascular tumor is very variable: ecchymotic, purpuric, inflammatory, or hemorrhagic. Its surface is smooth and shiny. On palpation, the tumor is hard and tender. Around and at a distance, petechiae and bruises may be present [1]. On the biological level, Thrombocytopenia is constant, often profound and associated with a decrease in the level of fibrinogen, a significant increase in D-dimers and fibrin degradation products, sometimes with iron deficiency anemia related to hemorrhagic syndrome or hemolytic microangiopathy [1], [2]. On the radiological level, an ultrasound assessment is useful to establish the diagnosis. The color Doppler confirms the diagnosis with a sensitivity of $84 \%$ and a specificity of $98 \%$. As for CT and MRI, they allow a better definition of these lesions, particularly for deep localizations [12], [13]. In MRI, these tumors appeared ill-defined, in isosignal T1, in hypersignal T2, diffusedly enhanced after injection of gadolinium and infiltrating the subcutaneous tissues [1]. Other explorations can help with diagnosis such as digital subtraction angiography, bone scintigraphy and standard radiology in search of regional osteolysis [1]. The guided echo biopsy makes it possible to differentiate the SKM from the other malignant neoplasias, however its realization is controversial, because of the important hemorrhagic and infectious risk on this ground [1], [13].

Therapeutically, publications usually relate to a single observation of SKM or a small series of cases. The effectiveness of a treatment is therefore difficult to establish by consensus. The therapeutic approach is multidisciplinary (onco-hematologists, dermatologists, radiologists and pediatric surgeons) [14, 15]. SKM treatment has two objectives: to control coagulopathy and to eradicate the vascular tumor.

The first objective aims to correct hematological anomalies, especially when an invasive procedure is planned or in the event of hemorrhage [15]. It can include the administration of fresh frozen plasma, cryoprecipitate, transfusion of red cells and platelets, the latter is however to be avoided because it stimulates the process and increases the risk of bleeding, with very rapid consumption in 1 to 24 hours infused platelets [16]. This phenomenon, which we have observed on several occasions, is confirmed in various observations in the literature, in particular that of Philips et al. Who report the worsening of an SKM treated by repeated platelet transfusions [17].

The treatment of the tumor is not codified, a consensus, published in 2013 recommended a dual therapy with vincristine $(0.05 \mathrm{mg} / \mathrm{kg})$ and corticosteroids $(2 \mathrm{mg} / \mathrm{kg} / \mathrm{d})$ from the start in case of complicated HEK of SKM [18]. In this study, out of 24 centers treating complicated SKM HEKs, 12 introduced this dual therapy straight away, 7 corticosteroids alone (29\%) and 2 vincristine alone (8\%). The use of INF in this context is based on its ability to inhibit the motility of the endothelial cells of the capillaries which gives it antiangiogenic activity [19]. The duration of treatment with vincristine is 21 weeks according to the recommendation of the Enjolras team $[1,20]$.

Side effects from INF include frequent reactions like fever, neutropenia, anemia, and others that are more rare and potentially more serious, such as liver cytolysis or spastic diplegia [21-22]. In the study by Michaud et al. [22] reporting the side effects of INF in pediatrics (69\% chronic hepatitis and 14\% vascular tumors), it appears that spastic diplegia was observed only in children treated before the age of 1 year. The inconsistent efficacy of conventional treatments and of INF as well as their side effects and the appearance of new, less restrictive therapies have definitively changed the place of INF in the management of SKM. Besides INF we find Propanolol, widely used in the treatment of childhood hemangioma (for its antivascularendothelialgrowth factor [VEGF] and anti-fibroblastgrowth factor [FGF] action) sometimes seems to be effective in taking charge of HEK and AT [23]. The benefit remains debated, the effect being variable depending on the series (36\% that of Chiu et al.) [24].

Sirolimus, an inhibitor of the essential transcription factor mamaliantarget of rapa-micyne (mTOR), used in our patient, has also been shown to be effective in the management of HEK resistant to corticosteroids and vincristine [25, 26]. Therapeutic dose is $0.1 \mathrm{mg} / \mathrm{kg} /$ day in two doses spaced 12 hours apart or $0.8 \mathrm{mg} / \mathrm{m} 2$ twice a day. The residual blood level should be between 5 and $15 \mathrm{ng} / \mathrm{ml}$. The side effects are dosedependent, combining diarrhea, dry mouth, peripheral edema and respiratory distress. The inhibition of 
mTOR, which is involved in many mechanisms of angiogenesis, makes this treatment very attractive for the management of resistant HEK. Other therapies such as bevacizumab (anti-VEGF monoclonal antibody used in oncology) have been used with success, but the results of these treatments remain inconstant [27, 28].

\section{Conclusion}

Physicians must be aware of the cause and effect relationship between vaccination and SKM so the diagnosis could be made quickly for optimal therapeutic management to improve survival and limit sequella. The capricious development and the absence of a standardized therapeutic protocol make it difficult to manage. The therapeutic decision must be multidisciplinary. It constantly balances the short-term vital prognosis and the undesirable effects of the treatments used. A better understanding of the pathophysiology of SKM could contribute to the development of new therapeutic pathways, in particular those involved in the inhibition of angiogenesis like SIROLIMUS used in our patient.

\section{References}

[1.] P. Le Nouail, V. Viseux and O. Enjolras et le groupe de recherche clinique en dermatologie pédiatrique, «Phénomène de Kasabach-Merritt », Ann DermatolVenereol, 134: 580-6, 2007.

[2.] O. Enjolras and M. Wassef, "Classification et données physiopathologiques en matière d'angiomes superficiels", Sang Thrombose Vaisseaux. Volume 10, Numéro 9, 563-71, 1998.

[3.] M.H. Sohn, H.J. Jeong, S.T. Lim, D.W. Kim and C.Y. Yim, "A Giant HepaticHemangiomaComplicated by Kasabach-Merritt Syndrome: Findings of Tc-99m RBC Scintigraphy and SPECT Including a Total Body Blood Pool Imaging Study", Nucl Med Mol Imaging, Vol. 43, No. 1, 2009.

[4.] J.Y. Tang, J. Chen, C. Pan, M.Z. Yin and M. Zhu, "Diffuse CavernousHemangioma of the spleen withKasabach-Merritt Syndrome misdiagnosed as idiopathicthrombocytopenia in a child, World J Pediatr, 4 (3): 227-230, 2008.

[5.] S. Kumar, B.Taneja, K.N. Saxena, N. Kalra. "Anaesthetic management of a neonatewithKasabachMerritt syndrome”, Indian J Anaesth, 57:292-4, 2013.

[6.] Maguiness S, Guenther L. Kasabach-Merritt Syndrome. J Cutan Med Surg 2002; 6:335-9.

[7.] Hall GW. Kasabach-Merritt syndrome: pathogenesis and management. Br J Haematol 2001; 112:851-62.

[8.] Seo SK, Suh JC, Na GY, Kim IS, Sohn KR. Kasabach-Merritt syndrome: identification of platelettrapping in a tuftedangioma by immunohistochemistry technique using monoclonal antibody to CD61. PediatrDermatol 1999; 16:392-4.

[9.] Gruman A, Liang MG, Mulliken JB, Fishman SJ, Burrows PE, Kozakewich HP, et al. Kaposiform hemangioendothelioma without kasabach-merritt phenomenon. $J$ Am Acad Dermatol 2005;52(4):616-22.

[10.] Woo CJ, Na JI, Hong JS, Hyo KS, Young BS, Hyun CK, et al. Intractable tufted angioma associated with kasabach-merritt syndrome. Ann Dermatol 2013;25 (1):129-30.

[11.] Croteau SE, Liang MG, Kozakewich HP, Alomari AI, Fishman SJ, Mulliken JB, et al. Kaposiform hemangioendothelioma: atypical features and risks of kasabach-merritt phenomenon en 107 referrals. J Pediatr 2013;162(1):142-7

[12.] M.M. Alhassan, I. Bassioni, A. Tikvica and A. Baderldeen." Large hepatichemangiomacomplicatedwithseverepolyhydramnios and kasabach-Merritt Syndrome" GynaecolPerinatol, 17(4):225-227, 2008.

[13.] R.V.W. Bach, R.V Carvalho, P.A.S Faria, A.R Gonçalves and C.H.V. Mariño, "Splenichaemangioma and Kasabach-Merritt Syndrome as differentialdiagnosis for abdominal mass in a newborn: case report », RevistaBrasileira de Cancerologia, 48(2): 247-251, 2002.

[14.] Maguiness S, Guenther L. Kasabach-Merritt Syndrome. J Cutan Med Surg 2002;6:335-9.

[15.] Hall GW. Kasabach-Merritt syndrome: pathogenesis and management. $\mathrm{Br} \mathrm{J}$ Haematol 2001;112:851-62.

[16.] Mulliken JB, Anupindi S, Ezekowitz RA, Mihm MC Jr. Case records of the Massachusetts General Hospital. Case 13-2004. A newborn girl with a large cutaneouslesion, thrombocytopenia, and anemia. N Eng J Med 2004;350:1764-75. 
[17.] Phillips WG, Mardsen JR. Kasabach-Merritt syndrome exacerbated by platelet transfusion. J R Soc Med 1993;86:231-2.

[18.] Drolet BA, Trenor 3rd. CC, Brandao LR, et al. Consensus-derivedpractice standards plan for complicatedKaposiformheman-gioendothelioma. J Pediatr 2013;163:285-91.

[19.] Caraglia M, Vitale G, Marra M, et al. Alpha-interferon and itseffects on signallingpathwayswithincells. CurrProteinPeptSci 2004;5:475-85.

[20.] Haisley-Royster C, Enjolras O, Frieden IJ, et al. Kasabach-merrittphenomenon: aretrospectivestudy of treatmentwithvincris-tine. J PediatrHematolOncol 2002;24:459-62.

[21.] Barlow CF, Priebe CJ, Mulliken JB, et al. Spasticdiplegia as acomplication of interferon Alfa-2a treatment of hemangiomasof infancy. J Pediatr 1998;132:527-30.

[22.] Michaud AP, Bauman NM, Burke DK, et al. Spasticdiplegia andothermotordisturbances in infants receivinginterferon-alpha.Laryngoscope 2004;114:1231-6.

[23.] Blatt J, McLean TW, Castellino SM, et al. A review of contem-porary options for medical management of hemangiomas,othervasculartumors, and vascular malformations. Pharmacol2013;139:327-33.

[24.] Chiu YE, Drolet BA, Blei F, et al. Variable response to propranololtreatment of kaposiformhemangioendothelioma, tuftedangi-oma, and Kasabach-Merritt phenomenon. Pediatr Blood Cancer2012;59:934-8.

[25.] Hammill AM, Wentzel M, Gupta A, et al. Sirolimus for thetreatment of complicatedvascular anomalies in children.Pediatr Blood Cancer 2011;57:1018-24.

[26.] Adam Z, Pour L, Krejci' M, et al. Successfultreatment ofangiomatosiswith thalidomide and interferon alpha. A de-scription of five cases and overview of treatment of angioma-tosis and proliferatinghemangiomas. Vnitr Lek 2010;56:810-23.

[27.] Agulnik M, Yarber JL, Okuno SH, et al. An open-label, multicen-ter, phase II study of bevacizumab for the treatment of angio-sarcoma and epithelioidhemangioendotheliomas. Ann Oncol2013;24:257-63.

[28.] Blatt J, Stavas J, Moats-Staats B, et al. Treatment of childhoodkaposiformhemangioendotheliomawithsirolimus. PediatrBlood Cancer 2010;55:1396-8. 